Doch viele Fragen, deren Beantwortung für die Zukunft entscheidend sein wird, konnten noch nicht beantwortet werden: Aus welchen Strukturen heraus konnten die Attentäter überhaupt ausgebildet und die Terroranschläge vorbereitet werden? Mit welchen zivilen und mit welchen militärischen Maßnahmen lassen sich ähnliche Attacken, wie die vom 11. September, verhindern? Über welche Optionen verfügen wir? Wie können die ungeheuren Spannungen im Nahen Osten und den angrenzenden Regionen, die auch Ausdruck einer sozialen Modernisierungskrise der islamischen Welt sind, in einen friedlichen Evolutionsprozess umgewandelt werden, der den Menschen eine konkrete Perspektive in der Welt des 21. Jahrhunderts eröffnet?

Wir müssen versuchen, Antworten auf diese Fragen zu entwickeln. Die Menschen erwarten dies von der Politik. Dabei landen wir ganz schnell bei der Weltordnung, die wir selber auf diesem Planeten zulassen. Das Nachfragen führt uns zu den globalen Tendenzen der Entstaatlichung, Privatisierung und Kommerzialisierung der Gewalt (Erhard Eppler), die uns längst in zahlreichen Weltregionen die größten Probleme bereiten. Dann müssen wir klären, ob es sich hierbei um die Auswirkungen einer Globalisierung handelt, die staatliche Gewalt schwächt, den Abstieg von Regionen und ganzen Subkontinenten (etwa des südlichen Afrika) schulterzuckend zur Kenntnis nimmt und bei der Reparaturfrage auf die Selbstheilungskräfte des Marktes setzt.

Die Zerbröselung des staatlichen Gewaltmonopols kennzeichnet heute keineswegs nur vergessene Drittweltregionen, dieses Geschwür wuchert längst in die fortgeschrittensten Industriestaaten hinein. Wo der Neoliberalismus solange gegen den Staat agiert hat, bis diesem die nötigen Mittel zur Aufrechterhaltung seines Gewaltmonopols verweigert werden, da entsteht eine neue Klassengesellschaft: Wer sich Sicherheit nicht kaufen kann, bleibt ohne Schutz und es entsteht jene »Zitadellengesellschaft«, die alle Konflikte und Probleme der gespaltenen Weltgesellschaft im überschaubaren Raum von Stadt und Land getreulich abbildet. Die alten Rezepte des Strukturkonservatismus, des Neoliberalismus oder gar des Populismus bleiben hier stumm oder führen geradewegs in die Sackgasse.

In der Welt von heute greifen Außen- und Innenpolitik genauso wie Sicherheits- und Entwicklungspolitik, Handels-, Finanzpolitik immer mehr ineinander. Und es ist noch nicht ausgemacht, wer sich die Definitionsmacht für diese Neue Zeit erobern wird. Dies allein ist Grund genug, für eine Fortsetzung von Rot-Grün über den 22. September hinaus zu kämpfen.

\title{
Überlegungen zur Zukunft der Transatlantischen Beziehungen ${ }^{1}$
}

Dr. Karl Lamers, MdB, außenpolitischer Sprecher der CDU/ CSU-Bundestagsfraktion, Berlin

1) Der Angriff des transnationalen Terrorismus vom 11. September 2001 auf die Symbole der militärischen und wirtschaftlichen Macht Amerikas - das Pentagon und das WTC - ist der radikalste Ausdruck der Auflehnung gegen die von den USA als dem Protagonisten des Westens geprägte Weltordnung. Er ist Zeichen ihrer tiefen Krise. Der Beifall für dieses monströse Ereignis bei den Massen in der islamischen Welt, aber auch Reaktionen in anderen ihrer Teile sind das eigentlich politisch Beunruhigende. Der Westen - nicht nur seine Vormacht Amerika - ist herausgefordert. Er muss eine grundlegende Neubewertung des Zustandes, in dem sich die Welt befindet, und eine Neubestimmung seiner Verantwortung für sie vornehmen, um die unerlässlichen militärischen und anderen operativen Maßnahmen in einen ebenso unerlässlichen, aber noch nicht vorhandenen, (definierten) politischen Rahmen stellen zu können. Der 11. September 2001 demonstriert die Verletzlichkeit der heutigen Zivilisation, und dass auch Amerika verwundbar

1 Rede am 27. Juni 2002. ist. Der Westen muss sich neu formieren und die tiefen Differenzen überwinden, die mit dem 11. September offenkundig wurden.

Der 11. September 2001 hat in brutaler Deutlichkeit die Notwendigkeit, ja die Unausweichlichkeit eines noch engeren europäisch-amerikanischen Zusammenwirkens gezeigt. $\mathrm{Zu}$ diesem Zweck müssen Europa und Amerika vorab und vor allem das ganze Ausmaß des Aufruhrs gegen die heutige Weltordnung und dessen Ursachen wahrnehmen, es sich bewusst machen, die Herausforderung annehmen, ihre Politik davon bestimmen lassen, alle Politik - die innere wie die äußere - und vor allem die Prioritäten anders setzen: Außenpolitik hat Vorrang, weil unser Wohl und Wehe, das der Völker des Westens, immer mehr vom Wohl und Wehe aller unserer Nachbarn abhängt und Nachbar sind heute alle.

Der konkrete Ausdruck der Prioritäten seiner Interessen ist der Haushalt eines Landes. In Deutschland sind die Ausgaben für auswärtige Zwecke, soweit sie in den Haushalten für das Auswärtige Amt, die Entwicklungshilfe und die Verteidigung zum Ausdruck kommen, seit 1990 bis 2002 von mehr als ca. 21 Prozent auf weniger als ca. 12 Prozent am Gesamthaushalt zurückgegangen. 
Unser Land setzt seine Prioritäten falsch. Wenn nach dem 11. September nichts mehr so wäre, wie es vorher war wie behauptet wurde -, dann müsste das in aller erster Linie für das politische Bewusstsein gelten. Davon aber ist in Deutschland so gut wie nichts zu spüren. Dasselbe gilt für Europa, sonst würde es spätestens jetzt mit einer Stimme sprechen.

2) Der Westen darf die Welt nicht nur unter dem Blickwinkel des Terrorismus sehen, darf nicht nur die Gefahren, sondern muss auch die Chancen für eine etwas bessere Welt wahrnehmen.

Wenige Wochen nach dem 11. September sagte ein radikaler pakistanischer Islamist von Amerika: »Das Land, das wir am meisten hassen, ist unser Paradies.«

Das zeigt die ganze Ambivalenz selbst der radikalsten Gegner der USA. Selbst sie sehnen sich nach gewissen Errungenschaften des Westens, weil nur sie ein besseres Leben versprechen. Der ganz überwiegende Teil der Menschheit weiß, dass er die Zusammenarbeit und die Hilfe des Westens braucht, und er will sie. Der Hass auf Amerika ist oft enttäuschte Hoffnung und Zuneigung. Noch hat der Westen die Mittel und die Chance, die Welt so zu gestalten, dass sie keine ihm feindselige wird. Die Völker, die nach einem besseren Leben, nach einem »Platz an der Sonne « streben, sind nicht seine »natürlichen Feinde $\ll$.

3) Der Westen darf nicht alles über den Kamm des Terrorismus scheren und nicht zulassen, dass seine Verbündeten es tun.

Es gilt, legitimen Widerstand auch mit gewaltsamen Mitteln gegen illegitime Herrschaft gegenüber illegitimem Terrorismus abzugrenzen. Der Westen selbst, vor allem die USA haben viele solche Widerstandsbewegungen in der Vergangenheit unterstützt. Die Mittel im Kampf gegen den Terrorismus dürfen ihn nicht fördern; die tieferen wirtschaftlichen, sozialen und politischen Ursachen müssen beseitigt werden, sollen nicht Terroristen wie Fische im Wasser schwimmen und dann unbesiegbar werden. Terrorismus - nationaler wie transnationaler - lässt sich nur besiegen, wenn zwei Elemente die Strategie bestimmen: Druck durch militärische, polizeiliche Maßnahmen und Isolierung innerhalb der eigenen Gesellschaft.

4) Der Westen darf den Kampf gegen den transnationalen Terrorismus sich nicht zur Feindschaft mit dem revolutionären Islam entwickeln lassen, um nicht die Flamme der Revolution anzufachen. Die teuflische Alternative, vor die Terrorismus uns stellen will, lautet: Kapitulation oder totale Feindschaft. In diese Falle dürfen wir nicht laufen. Polarisierung, Feindschaft, totaler Krieg sind die Antriebselemente von Al Qaida, mit ihnen wollen sie die Massen gewinnen; Demokratien sind ihnen wesensfremd und eine Bedrohung ihrer Eigenart.

5) Der Westen muss versuchen, die Welt und sich selbst mit den Augen der anderen zu sehen. Verstehen ist die erste Voraussetzung für Verständigung, Erkenntnis der eigenen Fehler für Lernen. Manichäismus führt zur totalen Feindschaft, die eben mit allen Mitteln verhindert werden muss.
6) Der Westen muss seine eigene ideelle Position in historischer Perspektive klären. Das Ergebnis ist, der Westen muss nicht seine Prinzipien ändern, sondern nach ihnen handeln, um seine Glaubwürdigkeit wiederherzustellen. Eine Lösung des Israel-Palästina-Konfliktes, die auch Palästinenser und Araber als gerecht empfänden, wäre dazu ein entscheidender Beitrag!

7) Der Westen muss eine Vorstellung von einer etwas besseren, etwas gerechteren, friedlicheren Weltordnung entwickeln. Ja, wir brauchen eine Vision von einer besseren Welt, um den Hass zu überwinden. Institutionell muss das eine Welt sein, die institutionalisiert zusammenarbeitet, das heißt, in der sich eine globale Rechtsordnung entwickelt. Bis dahin, und das heißt noch sehr lange hin, bedarf es einer Führungsnation, die nur die USA sein können. Die Richtung aber, in welche Amerika führt, muss eine Rechtsordnung sein. Dazu muss es bereit sein, sich selber auch dem Recht zu unterwerfen und Ausnahmen nur zu beanspruchen, wo sie sich zwingend seiner Rolle als Führungs- und Sanktionsmacht verdanken und nicht eigensüchtigen Interessen. Von der Weisheit, das heißt, wie maßvoll es diese Rolle spielt, hängt Entscheidendes für die Zukunft der Welt ab. Und das wiederum hängt entscheidend von dem Einfluss Europas auf Amerika ab. Auch deswegen muss der Westen sich neu formieren. Die NATO muss in ein Bündnis zwischen Amerika und Europa als handlungsfähige Einheit umgestaltet werden. Die EU als solche muss im NATO-Rat vertreten sein. Der politische Charakter der NATO muss ausgebaut werden, sie muss einen globalen Auftrag bekommen. Davon hängt die Zukunft der NATO $\mathrm{ab}$, nicht von der NATO-Osterweiterung. Eine Allianz, die sich nicht um die großen Fragen kümmert, hat keine große Zukunft. Entweder bleibt die NATO eine regionale Organisation zur sicherheitspolitischen Rückversicherung ihrer europäischen Mitglieder »für alle Fälle« und vor allem zur Absicherung der globalen Rolle der USA, oder sie wird selbst ein globaler Akteur, der es auch seinen europäischen Mitgliedern erlaubt, nicht nur den USA Hilfsdienste zu leisten, sondern die Welt mit zu gestalten. Ob dies gelingt, hängt von beiden Seiten $a b$, vor allen Dingen aber von den Europäern. Sie könnten schon heute von den Amerikanern Mitentscheidung bei ihrer Strategie gegen den transnationalen Terrorismus einfordern, weil sie mindestens in der Phase nach einer jeden militärischen Intervention - zur unerlässlichen Absicherung ihres Ergebnisses mit militärischen wie anderen Mitteln - gebraucht werden. Die Folgen von Entscheidungen automatisch mit $\mathrm{zu}$ tragen, die Amerika alleine getroffen hat, ist eine für Europa inakzeptable Arbeitsteilung. Erfolgversprechend wird diese Forderung aber natürlich nur sein, wenn Europa mit einer Stimme spricht und alle seine Kräfte, die militärischen wie die politischen, zusammenführt, mehr auf die Waagschale legt. (Die europäischen Länder müssen die Konsequenz aus der Tatsache ziehen, dass sie alle sogenannte große wie kleine - gestaltenden Einfluss nur noch im Verhältnis zueinander haben, in Bezug auf Länder also, die in einem besonderen Rechtsverhältnis zueinander stehen. Klassische Außenpolitik bezieht sich aber eben auf Länder, die ein solches nicht zueinander haben. In Bezug auf sie ist der Einfluss der europäischen Länder marginal, 
überspitzt formuliert, sie haben Außenbeziehungen, aber keine Außenpolitik.) Es muss uns $\mathrm{zu}$ denken geben, dass Europa in der kürzlichen strategischen Rede des US-Präsidenten in West Point, gehalten nur wenige Tage nach seinem Besuch im Reichstag, keine Rolle gespielt hat.

$\mathrm{Ob}$ Europa $\mathrm{zu}$ der notwendigen Einheit findet, ob es den Weg seiner Schicksalsgemeinschaft mit Amerika nur miterleidet oder ob es diesen auch mitgestaltet, darauf muss der soeben begonnene Verfassungsprozess eine Antwort finden. Sie wird aber nur gefunden werden, wenn es ein Einvernehmen über die Rolle Europas in der Welt, vor allem über sein Verhältnis zu Amerika, geben wird, und dieses wiederum hängt entscheidend von einem deutsch-französischen Einvernehmen ab. Das wiederum liegt keineswegs in erster Linie an Frankreich, wie es opinio communis in Deutschland zu sein scheint, denn wenn es Meinungsverschiedenheiten zwischen Amerika und Europa gibt, gibt es gewissermaßen drei idealtypische Positionen: eine britische als die Amerika nächste, eine französische als die gegenüber Amerika kritischste und die Deutschlands, das am liebsten nicht gefragt werden möchte. Frankreich und Deutschland müssen endlich eine gemeinsame Vorstellung vom Verhältnis Europas zur übrigen Welt, vor allem zu Amerika, entwickeln. Es gibt sie nicht, nicht ausreichend jedenfalls. Eine solche Vorstellung aber ist für das Selbstverständnis, für die Identität Europas, wichtiger als alles andere. Hätten wir sie, würde sich manch interner Zwist leichter beilegen.

Europa muss und kann nicht werden wollen wie Amerika, um ein selbständiger Akteur und ebenbürtiger Partner zu sein; das ist weder möglich noch wünschenswert, Europa muss vielmehr seine spezifischen Stärken entwickeln. Es muss nicht gleichartig wie, sondern gleichwertig mit den USA werden. Amerika seinerseits braucht einen Partner, der immer auch Widerlager sein muss. Grenzenlosigkeit führt immer zum Selbstverlust, auch grenzenlose Macht, was das Wort von Arthur M. Schlesinger erklärt: »Die amerikanische Außenpolitik schwankt zwischen Hybris und Fragmentierung.«

Europa muss in den Augen der nicht-westlichen Welt als eigenständiger Akteur innerhalb des Westens erscheinen und nicht nur als Anhängsel oder bestenfalls Juniorpartner der USA, sonst kann es die Rolle nicht spielen, welche von ihm zu Recht erwartet wird und die den Westen insgesamt stärkte. Es muss ein zuverlässiger Partner der Länder jenes Bereiches sein und ihre Sichtweise Amerika zu vermitteln versuchen, ohne sich gegen Amerika in Stellung bringen $\mathrm{zu}$ lassen, und es muss die Sichtweise Amerikas ihnen zu vermitteln versuchen.

Europa und Amerika haben die gleichen grundlegenden Interessen, weil sie die gleichen Ansichten vom Menschen und seinem Zusammenleben haben. Das ist das starke Fundament der transatlantischen Gemeinsamkeit, von der Präsident Bush zu Recht vor wenigen Wochen gesprochen hat. Und dennoch gehen die Meinungen, wie der Westen seine Stellung in der Welt verteidigen und gestalten soll, zwischen Europa und Amerika zunehmend auseinander. Im Mittelpunkt steht dabei die Rolle der militärischen Macht. Ob und inwieweit man über sie verfügt, bestimmt die Weltsicht wesentlich mit. Aber auch historische Erfahrungen tun das. Und das Lebensgefühl einer Gesellschaft, welche die erfolgreichste der Gegenwart ist, muss zwangsläufig zu dem Schluss kommen, dass das, was gut für Amerika ist, auch gut für die Welt sei.

Es ist hier nicht der Ort, um alle tieferen Gründe für alle Facetten der unterschiedlichen Lebensgefühle auf beiden Seiten des Atlantik zu analysieren, sondern nur um die Schlussfolgerung zu ziehen: Europa darf ihretwegen nicht immer stärkere Ressentiments entwickeln - also das Lebensgefühl dessen, der sich im Leben zu kurz gekommen vorkommt -, sondern die Ursachen für sie überwinden. Kritik an amerikanischer Politik ist zwar oft angebracht, aber solange Europa zwar weiß, was es nicht will, aber nicht, was es will, und so lange es auch keine ausreichenden Mittel hat oder sie nicht einsetzt, klingt seine Kritik hohl. Solange es sich damit begnügt, Amerika zu kritisieren, aber dann doch mit zu machen, wenn Amerika entschieden hat (wie das im Falle des Irak wahrscheinlich wieder so wäre), darf es seinen Vasallen-Status nicht beklagen.

Europa muss die Ursachen für seine Ressentiments gegenüber Amerika beseitigen: die zu große Abhängigkeit heute in globalen Fragen, wie früher in denen der europäischen Sicherheit, und das Gefühl, keinen angemessenen Einfluss auf Entscheidungen nehmen $\mathrm{zu}$ können, die es gleichwohl betreffen; keine unmittelbare Verantwortung und Entscheidungszwang zu haben. Einen solchen müssen die Europäer sich selber schaffen, um Unentschlossenheit und Uneinigkeit zu überwinden. Der Starke und Selbstbewusste hat keine Ressentiments; nur er ist ein verlässlicher Partner.

Europa hat keinen Grund, Ressentiments zu entwickeln. Es hat Stärken, über die Amerika nicht verfügt. Europa hat einen außerordentlichen Schatz an Erfahrungen in allen Teilen der Welt. Es hat aus seinen Niederlagen gelernt. Die nichtwestliche Welt erwartet geradezu sehnsüchtig ein stärkeres Engagement Europas auf allen Feldern der Politik, hat es doch alles, was dieser Teil der Menschheit braucht, ohne hegemonialer Absichten verdächtig oder überhaupt dazu fähig zu sein.

Schließlich meistert Europa etwas, was in der ganzen Welt und in vielen ihrer Regionen notwendig ist, soll der Frieden gesichert sein: die Entwicklung einer Rechtsordnung, der sich alle seine Mitglieder, freie und selbstbewusste Nationen, freiwillig unterwerfen, und es bemüht sich im Inneren, für seine Bürger eine Ordnung zu entwickeln, in der Freiheit und Solidarität gleichermaßen gelten.

Gerade dies ist das Wertvollste, was Europa vorzuweisen hat, was die europäischen Staaten in Bewahrung ihrer Vielfalt über Jahrzehnte geschaffen haben. Und diese Ordnung ist modern und zielweisend.

Amerika bleibt ein faszinierendes Land - gerade wegen seines ganz eigenen Charakters. Und die Amerikaner haben im Umgang mit den furchtbaren Ereignissen des 11. September gezeigt, dass sie wahrlich Charakter haben. 
Amerika muss bereit sein, das Wesen Europas zu verstehen und damit seine Modernität zu begreifen. Es muss in seinem eigensten Interesse bereit sein, Europa nicht nur als militärischen oder politischen Verbündeten oder als wirtschaftlichen Mitstreiter zu sehen, sondern als echten Partner mit dem ihm eigenen Charakter. Die Amerikaner werden dies verstehen, wenn sie es verstehen müssen, und wenn wir Europäer mehr in die Waagschale zu werfen bereit sind.

Die USA sind der mächtigste Akteur in der heutigen Welt Europa ist der modernste. »Europa ist ein Beitrag zu einer besseren Welt.« Nichts ist vordringlicher, als dieses Wort Jean Monnets stärker - sehr viel stärker - zur Geltung zu bringen.

\section{Kurs halten unter schweren Bedingungen: Bilanz und Perspektiven grüner Sicherheits- und Friedenspolitik}

\author{
Winfried Nachtwei, MdB, Bünd- \\ nis 90/Die Grünen, Mitglied \\ des Verteidigungsausschusses \\ und des Unterausschusses $A b$ - \\ rüstung, Rüstungskontrolle und \\ Nichtverbreitung, Berlin
}

»Deutsche Außenpolitik ist Friedenspolitik « behaupteten SPD und Bündnis 90/Die Grünen im Oktober 1998 in ihrer Koalitionsvereinbarung voller friedenspolitischer Selbstgewissheit. Sie versprachen, »sich mit aller Kraft um die Entwicklung und Anwendung von wirksamen Strategien und Instrumenten der Krisenprävention und der friedlichen Konfliktregelung zu bemühen« und dafür eine Infrastruktur aufzubauen.

Vier Jahre später kann man festhalten, dass sich Deutschland unter Rot-Grün in der Tat zu einem international anerkannten und vielfach treibenden Friedensakteur entwickelt hat. Wenn es darum ging, Handlungsspielräume friedensförderlich $\mathrm{zu}$ nutzen, hat der grüne Faktor sicher eine weit über die 6,7\% hinausragende Rolle gespielt. Doch während im Ausland die Leistungen der deutschen Außen- und Friedenspolitik positiv gewürdigt werden, scheiden sich daran in Deutschland die Geister. Unter Rot-Grün beteiligte sich das demokratische Deutschland erstmalig an zwei Kriegseinsätzen. Die Auslandseinsätze der Bundeswehr wuchsen in Dimensionen, wie sie 1998 kaum jemand für möglich gehalten hätte.

Kein Wunder, dass gerade bei Friedensbewegten, bei Teilen der Friedensforschung und der rot-grünen Anhängerschaft die Enttäuschung tief sitzt und die Urteile hart sind. Rot-Grün habe die Kultur der militärischen Zurückhaltung aufgegeben und das Militär zum normalen Instrument deutscher Außenpolitik gemacht, ja, Rot-Grün treibe vor allem Kriegspolitik im machtpolitischen Interesse. Vor allem den Grünen hängt dabei der Vorwurf an, ihre pazifistischen Prinzipien verraten zu haben.

Auf der anderen Seite wirft die bürgerliche Opposition Rot-Grün vor, die Bundeswehr kaputt zu sparen und damit die internationale Position der Bundesrepublik, ja, ihre Sicherheit zu gefährden.
Jenseits dieser Pole lautstarker Kritik findet rot-grüne Außenund Sicherheitspolitik zugleich breite Zustimmung über Parteigrenzen hinweg. Ihr wird zugute gehalten, in verantwortlicher Weise und als verlässlicher Partner zur internationalen Sicherheit beizutragen. Die Spitzen-Popularitätswerte für Joschka Fischer sind dafür ein Indikator.

Die folgenden Ausführungen sind unvollständig. Sie konzentrieren sich auf die Fallbeispiele Balkan und Terrorismus/Afghanistan sowie den Aufbau sicherheits- und friedenspolitischer Fähigkeiten (Krisenprävention und Bundeswehrreform).

\section{Bühnenwechsel}

Beide Koalitionsparteien waren unzureichend auf die außenund sicherheitspolitische Regierungsverantwortung vorbereitet. Vor allem in der Militärfrage bestand keine Klarheit und Einigkeit in und zwischen den beiden Parteien. Die Bündnisgrünen hatten im Kontext der Balkankriege und in einem schmerzhaften Erfahrungs- und Lernprozess wohl schrittweise ihre Haltung zu Gewaltfreiheit und Militär geändert, so dass die Bundestagsfraktion erstmalig am 19. Juni 1998 einem Auslandseinsatz der Bundeswehr (SFOR) zustimmte. In ihrem Wahlprogramm hatten sie aber noch wenig Rücksicht auf die Realitäten von Regierungsverantwortung genommen. Damit waren ab Koalitionsbeginn abrupte politische Schwenks einerseits und tiefe Enttäuschungen andererseits regelrecht vorprogrammiert.

Schon die Koalitionsvereinbarung verlangte Bekenntnisse zur NATO, zur Werte- und Interessengemeinschaft mit den USA und zur Bundeswehr, die der Einstellung vieler Grüner zuwiderliefen. Sehr schnell bekamen die Regierungsgrünen zu spüren, dass der Wechsel von der Opposition in die Regierung nicht ein Rollen-, sondern ein kompletter Bühnenwechsel war: Der »Spielplan« ist zu erheblichen Teilen fremdbestimmt; deutsche Außenpolitik agiert immer im multilateralen 